\title{
DIGITALCOMMONS
}

$@$ WAYNESTATE-

Wayne State University

$12-5-2003$

\section{Equilibrium Problems with Equilibrium Constraints via Multiobjective Optimization}

Boris S. Mordukhovich

WayneState University, boris@math.wayne.edu

\section{Recommended Citation}

Mordukhovich, Boris S., "Equilibrium Problems with Equilibrium Constraints via Multiobjective Optimization" (2003). Mathematics Research Reports. Paper 21.

http://digitalcommons.wayne.edu/math_reports/21

This Technical Report is brought to you for free and open access by the Mathematics at DigitalCommons@WayneState. It has been accepted for inclusion in Mathematics Research Reports by an authorized administrator of DigitalCommons@WayneState. 


\section{EQUILIBRIUM PROBLEMS WITH EQUILIBRIUM CONSTRAINTS VIA MULTIOBJECTIVE OPTIMIZATION}

BORIS S. MORDUKHOVICH

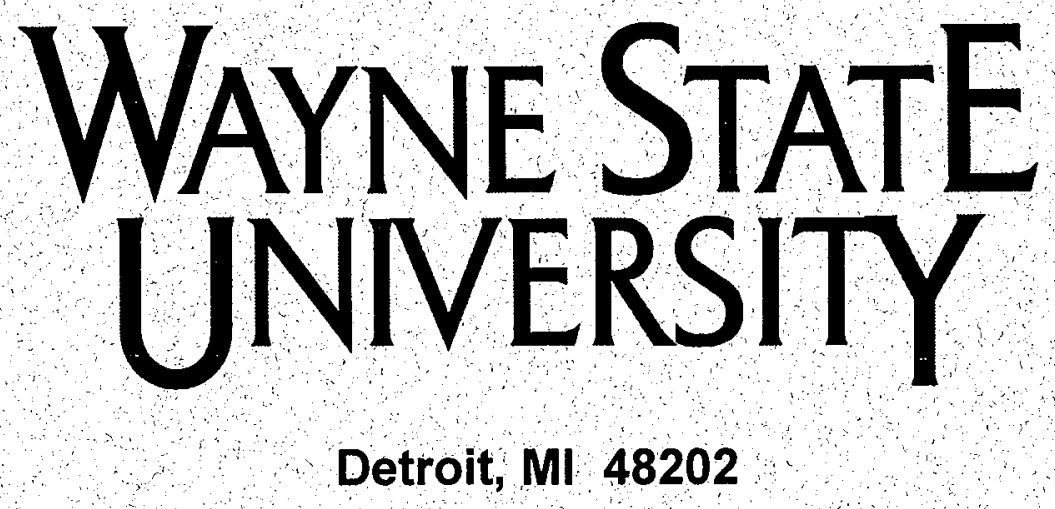

Department of Mathematics

Research Report

2003 Series

\#17 


\title{
EQUILIBRIUM PROBLEMS WITH EQUILIBRIUM CONSTRAINTS VIA MULTIOBJECTIVE OPTIMIZATION ${ }^{1}$
}

\author{
Boris S. Mordukhovich \\ Department of Mathematics Wayne State University, Detroit, MI 48202 \\ boris@math.wayne.edu \\ Dedicated to Olvi Mangasarian on occasion of his 70th birthday
}

\begin{abstract}
The paper concerns a new class of optimization-related problems called Equilibrium Problems with Equilibrium Constraints (EPECs). One may treat them as two level hierarchical problems, which involve equilibria at both lower and upper levels. Such problems naturally appear in various applications providing an equilibrium counterpart (at the upper level) of Mathematical Programs with Equilibrium Constraints (MPECs). We develop a unified approach to both EPECs and MPECs from the viewpoint of multiobjective optimization subject to equilibrium constraints. The problems of this type are intrinsically nonsmooth and require the use of generalized differentiation for their analysis and applications. This paper presents necessary optimality conditions for EPECs in finite-dimensional spaces based an advanced generalized variational tools of variational analysis. The optimality conditions are derived in normal form under certain qualification requirements, which can be regarded as proper analogs of the classical Mangasarian-Fromovitz constraint qualification in the general settings under consideration.
\end{abstract}

Keywords: Equilibrium Problems with Equilibrium Constraints, Multiobjective Optimization, Necessary Optimality Conditions, Variational Analysis, Generalized Differentiation.

\section{Introduction}

There is a growing interest to studying problems of hierarchical optimization, where sets of feasible solutions to upper level problems arise as sets of optimal solutions, or equilibrium points, to some lower level problems. A large class of such hierarchical problems has been unified in [5] as Mathematical Programs with Equilibrium Constraints (MPECs). A characteristic feature of these nonstandard problems of mathematical programming is the presence, among other constraints, "equilibrium constraints" of the type $y \in S(x)$, where the sets $S(x)$ often represent solution maps to some parametric variational inequality or complementarity problem. MPECs frequently appear in various aspects of hierarchical optimization and equilibrium theory as well as in many practical applications, especially to mechanical and economic modeling. Such problems are intrinsically nonsmooth and require tools of generalized differentiation for their analysis and applications. We refer the reader to the books $[3,5,18]$ for systematic expositions, examples, and applications of various MPECs and also to the papers $[11,16,22,23]$ for first-order necessary optimality conditions and related topics.

Quite recently an attention of researchers and practitioners has been drawn to hierarchical models that contains equilibrium problems at both lower and upper levels; see $[4,17]$ for examples and discussions. Such problems are naturally called Equilibrium Problems with Equilibrium Constraints

\footnotetext{
${ }^{1}$ Research was partly supported by the National Science Foundation under grants DMS-0072179 and DMS-0304989
} 
(EPECs). They are different from MPECs in that instead of minimizing real-valued functions under equilibrium constraints, EPECs involve some equilibrium criteria at the upper level.

It seems naturally to unify both MPECs and EPECs from the viewpoint of multiobjective optimization with equilibrium constraints. We have briefly addressed these issues in the note [12], which contains some necessary optimality conditions for such problems based on advanced generalized differentiation tools of variational analysis. The present paper is an extended version of [12], with proofs, more results, and discussions. Here, as in [12], we concern EPECs and MPECs in finite-dimensional spaces. Similar results are also available in infinite dimensions, but the proofs and even formulations are much more involved. We refer the reader to the forthcoming book [13] for detailed treatment of both finite-dimensional and infinite-dimensional problems. The case of MPECs is considered in [11], where some results are very specific for real-valued cost functions.

In what follows we develop two approaches to multiobjective optimization problems with equilibrium constraints, where optimal solutions at the upper level are understood either in the sense of optimization of a vector function with respect to a certain generalized order defined by a given subset of the range space, or with respect to some closed preference relation. Both approaches, which lead to generally different results, cover typical equilibrium problems at the upper level, and so the first-order necessary optimality conditions obtained in both ways are well applied to EPECs. To derive such conditions for problems with equilibrium constraints coming from the lower level, we employ generalized differential calculus of modern variational analysis, including chain rules for second-order subdifferentials that play a major role in the analysis of MPECs and EPECs. The necessary optimality conditions obtained in both ways, as well as the calculus rules for generalized differentiation, are based on the extremal principle for systems of sets and set-valued mappings, which can be viewed as a variational counterpart of the convex separation principle in nonconvex settings; see $[7,8,15]$ for more details and references.

The paper is organized as follows. In Section 2 we review the basic tools of generalized differentiation used below for deriving necessary optimality conditions. Section 3 is devoted to the generalized order optimality in multiobjective problems and to necessary optimality conditions for EPECs obtained in this way. Section 4 presents necessary optimality conditions for EPECs derived from the analysis of multiobjective optimization with respect to closed preference relations.

Our notation is basically standard. Recall that the Painlevé-Kuratowski upper/outer limit of a set-valued mapping $F: \mathbb{R}^{n} \Rightarrow \mathbb{R}^{m}$ at a point $\bar{x}$ is defined by

$$
\operatorname{Limsup}_{x \rightarrow \bar{x}} F(x):=\left\{y \in \mathbb{R}^{m} \mid \exists x_{k} \rightarrow \bar{x}, \exists y_{k} \rightarrow y \text { with } y_{k} \in F\left(x_{k}\right) \text { as } k \rightarrow \infty\right\} \text {. }
$$

Recall also that $x \stackrel{\Omega}{\rightarrow} \bar{x}$ and $x \stackrel{\varphi}{\rightarrow} \bar{x}$ mean that $x \rightarrow \bar{x}$ with $x \in \Omega$ and that $x \rightarrow \bar{x}$ with $\varphi(x) \rightarrow \varphi(\bar{x})$ for sets $\Omega \subset X$ and functions $\varphi: X \rightarrow \overline{\mathbb{R}}:=[-\infty, \infty]$, respectively.

\section{Generalized Differentiation}

In this section we define and review the basic generalized differentiation constructions used in the paper. They were introduced earlier by the author and then have been developed and employed in many publications; see, e.g., $[6,7,21]$ for more details and references. Let us start with generalized normals to sets. 
Given $\Omega \subset \mathbb{R}^{n}$ and $\vec{x} \in \Omega$, the normal cone to $\Omega$ at $\bar{x}$ is defined by

$$
N(\bar{x} ; \Omega):=\operatorname{Limsup}_{x \rightarrow \bar{x}}[\operatorname{cone}(x-\Pi(x ; \Omega))],
$$

where "cone" stands for the conic hull of a set and where $\Pi(\cdot ; \Omega)$ denoted the Euclidean projector of $x$ to the closure $\operatorname{cl} \Omega$, i.e.,

$$
\Pi(x ; \Omega):=\{w \in \operatorname{cl} \Omega \mid\|x-w\|=\operatorname{dist}(x ; \Omega)\} .
$$

For convex sets this cone reduces to the normal cone of convex analysis, but it is generally nonconvex even in simple settings, e.g., for the epigraphical and graphical sets associated with nonsmooth real functions as $\Omega=\operatorname{epi}(-|x|)$ and $\Omega=\operatorname{gph}|x|$. Note that the Clarke normal cone to $\Omega$ at $\bar{x}$ agrees with the convex closure to $N(\bar{x} ; \Omega)$; in particular, it is the whole space $R^{2}$ for the second set above. The basic normal cone (2.1) can be equivalently represented as

$$
N(\bar{x} ; \Omega)=\underset{x \rightarrow \bar{x}}{\operatorname{Limsup}} \widehat{N}(x ; \Omega)
$$

where the prenormal, or Fréchet normal, cone $\hat{N}(\cdot ; \Omega)$ is defined by

$$
\widehat{N}(\bar{x} ; \Omega):=\left\{x^{*} \in R^{n} \mid \limsup _{\substack{\Omega \rightarrow \\ \rightarrow}} \frac{\left\langle x^{*}, x-\bar{x}\right\rangle}{\|x-\bar{x}\|} \leq 0\right\} \text { for } \bar{x} \in \Omega
$$

and by $\widehat{N}(\bar{x} ; \Omega)=\emptyset$ for $\bar{x} \notin \Omega$. Note that the prenormal cone $\widehat{N}(\bar{x} ; \Omega)$ is the polar/dual cone (in finite dimensions!) to the (Bouligand) contingent cone well known in the optimization theory, while the basic normal cone $N(\bar{x} ; \Omega)$ cannot be dual to any tangent cone due to its nonconvexity.

Given a set-valued mapping $F: \mathbb{R}^{n} \rightrightarrows \mathbb{R}^{m}$ and a point $(\bar{x}, \bar{y})$ from its graph

$$
\operatorname{gph} F:=\{(x, y) \mid y \in F(x)\},
$$

the coderivative $D^{*} F(\bar{x}, \bar{y}): \mathbb{R}^{m} \rightrightarrows \mathbb{R}^{n}$ of $F$ at $(\bar{x}, \bar{y})$ is defined by

$$
D^{*} F(\bar{x}, \bar{y})\left(y^{*}\right):=\left\{x^{*} \in \mathbb{R}^{n} \mid\left(x^{*},-y^{*}\right) \in N((\bar{x}, \bar{y}) ; \operatorname{gph} F)\right\} .
$$

In general, $D^{*} F(\bar{x}, \bar{y})(\cdot)$ is a positively homogeneous mapping that reduces to the adjoint Jacobian

$$
D^{*} f(\bar{x})\left(y^{*}\right)=\left\{\nabla f(\bar{x})^{*} y^{*}\right\}, \quad \bar{y}=f(\bar{x}), \quad y^{*} \in \mathbb{R}^{m}
$$

when $F=f: \mathbb{R}^{n} \rightarrow \mathbb{R}^{m}$ is single-valued and strictly differentiable at $\bar{x}$, which is automatic for locally smooth $\left(\mathcal{C}^{1}\right)$ mappings.

Given an extended-real-valued function $\varphi: \mathbb{R}^{n} \rightarrow \overline{\mathbb{R}}$ finite at $\bar{x}$, we define its basic subdifferential $\partial \varphi$ and singular subdifferential $\partial^{\infty} \varphi$ at this point by

$$
\partial \varphi(\bar{x}):=D^{*} E_{\varphi}(\bar{x}, \varphi(\bar{x}))(1) \text { and } \partial^{\infty} \varphi(\bar{x}):=D^{*} E_{\varphi}(\bar{x}, \varphi(\bar{x}))(0)
$$

via the coderivative of the epigraphical multifunction $E_{\varphi}(x):=\{\mu \in \mathbb{R} \mid \mu \geq \varphi(\bar{x})\}$. There are various equivalent descriptions of both subdifferentials in (2.3) for lower semicontinuous (l.s.c.) functions given by

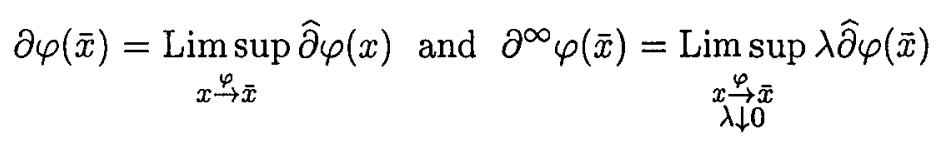


via the Fréchet, or regular, subdifferential defined by

$$
\widehat{\partial} \varphi(\bar{x}):=\left\{x^{*} \in \mathbb{R}^{n} \mid \liminf _{x \rightarrow \bar{x}} \frac{\varphi(x)-\varphi(\bar{x})-\left\langle x^{*}, x-\bar{x}\right\rangle}{\|x-\bar{x}\|} \geq 0\right\} .
$$

Note that $\partial^{\infty} \varphi(\bar{x})=\{0\}$ and $\partial \varphi(\bar{x}) \neq \emptyset$ if $\varphi$ is Lipschitz continuous around $\bar{x}$, and that one has the scalarization formula

$$
D^{*} f(\bar{x})\left(y^{*}\right)=\partial\left\langle y^{*}, f\right\rangle(\bar{x}) \neq \emptyset \text { for all } y^{*} \in \mathbb{R}^{m}
$$

when the mapping $f: \mathbb{R}^{n} \rightarrow \mathbb{R}^{m}$ is single-valued and locally Lipschitzian around this point.

Given $\varphi: \mathbb{R}^{n} \rightarrow \overline{\mathbb{R}}$ and $(\bar{x}, \bar{y}) \in \operatorname{gph} \partial \varphi$, we define the second-order subdifferential of $\varphi$ at $\bar{x}$ relative to $\bar{y}$ as the coderivative of the first-order subdifferential mapping:

$$
\partial^{2} \varphi(\bar{x}, \bar{y})(u):=D^{*}(\partial \varphi)(\vec{x}, \bar{y})(u), \quad u \in \mathbb{R}^{n} .
$$

Observe that for twice continuously differentiable functions $\varphi$ the second-order construction (2.4) reduces to the Hessian matrix

$$
\partial^{2} \varphi(\bar{x})(u)=\left\{\nabla^{2} \varphi(\bar{x}) u\right\} \text { with } \nabla^{2} \varphi(\bar{x})^{*}=\nabla^{2} \varphi(\bar{x})
$$

Among the most important advantages of our basic tools (2.1)-(2.4) in comparison with other generalized differential constructions of nonsmooth variational analysis, there is a comprehensive calculus allowing one to compute and estimate normals, subgradients, and coderivatives of various compositions, and also complete coderivative characterizations of metric regularity, openness, and Lipschitzian properties of multifunctions that play a fundamental role in many aspects of nonlinear analysis, especially those related to optimization, stability, and equilibrium; see the books $[6,21]$ and the recent papers $[9,14]$ for more details and references.

\section{Necessary Conditions for EPECs via Generalized Order Opti- mality}

In this section we consider multiobjective problems with equilibrium constraints whose optimal solutions are understood with respect to the following concept of generalized order optimality that particularly includes conventional notions of efficiency and equilibrium in various problems of vector optimization.

Definition 3.1 (generalized order optimality). Given a mapping $f: \mathbb{R}^{n} \rightarrow \mathbb{R}^{d}$ and a set $\Theta \subset \mathbb{R}^{d}$ containing the origin, we say that a point $\bar{x} \in X$ is LOCALLY $(f, \Theta)$-OPTIMAL if there are a neighborhood $U$ of $\bar{x}$ and a sequence $\left\{z_{k}\right\} \subset \mathbb{R}^{d}$ with $\left\|z_{k}\right\| \rightarrow 0$ as $k \rightarrow \infty$ such that

$$
f(x)-f(\bar{x}) \notin \Theta-z_{k} \quad \text { for all } \quad x \in U \text { and } k \in \mathbb{I}:=\{1,2, \ldots\} \text {. }
$$

The set $\Theta$ in Definition 3.1 generates an order/preference relation between $z_{1}, z_{2} \in \mathbb{R}^{d}$ defined via $z_{1}-z_{2} \in \Theta$. In the scalar case of $d=1$ and $\Theta=\mathbb{R}$ - the above optimality notion clearly reduces to the standard local optimality. Note that we do not generally assume that $\Theta$ is either convex or its interior is nonempty. If $\Theta$ is a convex subcone of $\mathbb{R}^{d}$, then the above optimality concept covers 
the conventional notion of optimality requiring that there is no $x \in U$ with $f(x)-f(\bar{x}) \in \operatorname{ri} \Theta$. This relates to weak Pareto optimality (efficiency, equilibrium) and is sometimes called Slater optimality. To reduce this notion to the one in Definition 3.1, it suffices to take $z_{k}:=-z_{0} / k$ for $k \in \mathbb{N}$ in (3.1) with some $z_{0} \in \mathrm{ri} \Theta$. The standard notion of Pareto optimality can be formulated in these terms as the absence of $x \in U$ for which $f(x)-f(\bar{x}) \in \Theta$ and $f(\bar{x})-f(x) \notin \Theta$. Of course, the Pareto-type notions can be written in the classical terms of utility functions when $\Theta=\mathbb{R}_{-}^{m}$.

Another example of the generalized order $(f, \Theta)$-optimality is given by the minimax criterion

$$
\min _{x \in \mathbb{R}^{n}} \max _{z^{*} \in \Lambda}\left\langle z^{*}, f(x)\right\rangle
$$

over a compact set $\Lambda \subset \mathbb{R}^{d}$ provided that there is $z_{0} \in \mathbb{R}^{d}$ with $\left\langle z^{*}, z_{0}\right\rangle>0$ for all $z^{*} \in \mathbb{R}^{d}$. Indeed, a local solution $\bar{x}$ to the above minimax problem easily reduces to the $(f, \Theta)$-optimality with

$$
\Theta:=\left\{z \in \mathbb{R}^{d} \mid\left\langle z^{*}, z\right\rangle \leq 0 \text { whenever } z^{*} \in \mathbb{R}^{d}\right\}
$$

by taking $z_{k}:=z_{0} / k$ in Definition 3.1 and assuming for simplicity that $\max _{z^{*} \in \Lambda}\left\langle z^{*}, f(\vec{x})\right\rangle=0$.

To derive necessary optimality conditions for EPECs, let us first consider local $(f, \Theta)$-optimal points of bivariate vector functions $f(x, y)$ subject to abstract equilibrium constraints in the form $y \in S(x)$, where $S: \mathbb{R}^{n} \rightrightarrows \mathbb{R}^{m}$ is an arbitrary set-valued mapping. In this context $S(x)$ may be a collection of equilibrium points (or optimal solutions to a lower-level problem) depending on the parameter $x$, while $y$ is the decision variable in the upper level optimization/equilibrium problem over $y \in S(x)$. The following theorem gives necessary conditions for $(f, \Theta)$-optimal solutions $(\bar{x}, \bar{y})$ under abstract equilibrium constraints.

Theorem 3.2 (generalized order optimality subject to abstract equilibrium constraints). Let $(\bar{x}, \bar{y}) \in \operatorname{gph} S$ be locally $(f, \Theta)$-optimal subject to $y \in S(x)$, where $f: \mathbb{R}^{n} \times \mathbb{R}^{m} \rightarrow \mathbb{R}^{d}$ with $\bar{z}:=f(\bar{x}, \bar{y})$, where $\Theta \subset \mathbb{R}^{d}$ with $0 \in \Theta$, and where $S: \mathbb{R}^{n} \rightrightarrows \mathbb{R}^{m}$. Then there is $z^{*} \in \mathbb{R}^{d}$ satisfying

$$
\left(0,-z^{*}\right) \in N((\tilde{x}, \bar{y}, \bar{z}) ; \mathcal{E}(f, S, \Theta)), \quad z^{*} \in N(0, \Theta) \backslash\{0\}
$$

provided that the "generalized epigraphical" set

$$
\mathcal{E}(f, S, \Theta):=\{(x, y, z) \in X \times Y \times Z \mid f(x, y)-z \in \Theta, y \in S(x)\}
$$

is locally closed around $(\bar{x}, \bar{y}, \bar{z})$. The latter yields

$$
0 \in D^{*} f(\bar{x}, \bar{y})\left(z^{*}\right)+N((\bar{x}, \bar{y}) ; \operatorname{gph} S), \quad z^{*} \in N(0, \Theta) \backslash\{0\}
$$

if $f$ is continuous around $(\bar{x}, \bar{y}), \Theta$ is locally closed around 0 , and the qualification condition

$$
\left[\left(x^{*}, y^{*}\right) \in D^{*} f(\bar{x}, \bar{y})(0), \quad-x^{*} \in D^{*} S(\bar{x}, \bar{y})\left(y^{*}\right)\right] \Longrightarrow x^{*}=y^{*}=0
$$

is fulfilled. Moreover, (3.4) holds automatically and (3.2) is equivalent to

$$
0 \in \partial\left\langle z^{*}, f\right\rangle(\bar{x}, \bar{y})+N((\bar{x}, \bar{y}) ; \operatorname{gph} S), \quad z^{*} \in N(0, \Theta) \backslash\{0\}
$$

if $f$ is Lipschitz continuous around $(\bar{x}, \bar{y})$. 
Proof. First observe that the abstract. EPEC under consideration is equivalent to the following multiobjective optimization problem under geometric constraints: find a local $(f, \Theta)$-optimal point $(\bar{x}, \bar{y})$ subject to $(\bar{x}, \bar{y}) \in \operatorname{gph} S$. It is easy to check that $(\bar{x}, \bar{y}, \bar{z})$ is an extremal point in sense of $[7$, Definition 3.1] of the system of two locally closed sets

$$
\Omega_{1}:=\mathcal{E}(f, S, \Theta) \text { and } \quad \Omega_{2}:=\operatorname{cl} U \times\{\bar{z}\}
$$

in the space $R^{n} \times \mathbb{R}^{m} \times \mathbb{R}^{d}$, where $U$ is a neighborhood of $(\bar{x}, \bar{y})$ from Definition 3.1. Applying the extremal principle from [7, Theorem 3.2] to the set system $\left\{\Omega_{1}, \Omega_{2}\right\}$ above, we immediately arrive at (3.2). Next observe that the set $\mathcal{E}(f, S, \Theta)$ is represented as the inverse image

$$
\mathcal{E}(f, S, \Theta)=g^{-1}(\Theta) \text { with } g(x, y, z):=f(x, y)+\Delta((x, y) ; g p h S)-z
$$

where $\Delta(u ; \Omega)=0 \in \mathbb{R}^{d}$ for $u \in \Omega \subset X \times Y$ and $\Delta(u ; \Omega)=\emptyset$ otherwise. Applying now the calculus rules from [7, Corollaries 4.5 and 5.5] under the qualification condition (3.4), we derive (3.3) from (3.2). The last optimality condition under the Lipschitz continuity of $f$ follows directly from the scalarization formula of Section 2, which also ensures the fulfillment of the qualification condition (3.4) and completes the proof of the theorem.

Next we consider a general class of equilibrium constraints typical for MPECs and EPECs. They are described by the parametric variational systems

$$
0 \in q(x, y)+Q(x, y)
$$

where $q: \mathbb{R}^{n} \times \mathbb{R}^{m} \rightarrow \mathbb{R}^{p}$ and $Q: \mathbb{R}^{n} \times \mathbb{R}^{m} \rightrightarrows \mathbb{R}^{p}$ are, single-valued and set-valued mappings called for simplicity by base and field, respectively. This is actually the classical format of generalized equations in the sense of Robinson [19], where we now allow the dependence on the parameter $x$ not only of the base $f$ but of the field $Q$ as well; cf. also [20]. It is well known that model (3.5) covers a vast majority of variational systems important in applications. In particular, (3.5) reduces to the parametric variational inequality

$$
\text { find } y \in \Omega \text { such that }\langle q(x, y), u-y\rangle \geq 0 \text { for all } u \in \Omega
$$

when $Q(y)=N(y ; \Omega)$ is the normal cone mapping generated by a convex set $\Omega \subset \mathbb{R}^{m}$. This gives the classical nonlinear complementarity problem when $\Omega=\mathbb{R}_{+}^{m}$.

The next theorem provides necessary conditions for generalized order optimality subject to the equilibrium constraints (3.5). For simplicity we present results only in the case of locally Lipschitzian mappings $f$ and $q$; the general case of continuous mappings $f$ and $q$ is available in [13], where the results are expressed in the coderivative terms for these mappings.

Theorem 3.3 (generalized order optimality subject to equilibrium constraints governed by variational systems). Let $(\bar{x}, \bar{y})$ be locally $(f, \Theta)$-optimal subject to the equilibrium constraints (3.5), where $f: \mathbb{R}^{n} \times \mathbb{R}^{m} \rightarrow \mathbb{R}^{d}$ and $q: \mathbb{R}^{n} \times \mathbb{R}^{m} \rightarrow \mathbb{R}^{p}$ are Lipschitz continuous around $(\vec{x}, \bar{y})$ with $\bar{p}:=-q(\bar{x}, \bar{y})$, and where $\Theta \subset \mathbb{R}^{d}$ and $\operatorname{gph} Q \subset \mathbb{R}^{n} \times \mathbb{R}^{m} \times \mathbb{R}^{p}$ are locally closed around $0 \in \Theta$ and $(\bar{x}, \bar{y}, \bar{p})$, respectively. Assume that the adjoint generalized equation

$$
0 \in \partial\left\langle p^{*}, q\right\rangle(\bar{x}, \bar{y})+D^{*} Q(\bar{x}, \bar{y}, \bar{p})\left(p^{*}\right)
$$


has only the trivial solution $p^{*}=0$. Then there are $z^{*} \in N(0 ; \Theta) \backslash\{0\}$ and $p^{*} \in \mathbb{R}^{p}$ such that

$$
0 \in \partial\left\langle z^{*}, f\right\rangle(\bar{x}, \bar{y})+\partial\left\langle p^{*}, q\right\rangle(\bar{x}, \bar{y})+D^{*} Q(\bar{x}, \bar{y}, \bar{p})\left(p^{*}\right) .
$$

Proof. This follows from Theorem 3.2 with

$$
S(x):=\left\{y \in \mathbb{R}^{m} \mid 0 \in q(x, y)+Q(x, y)\right\}
$$

due to the coderivative inclusion

$$
D^{*} S(\bar{x}, \bar{y})\left(y^{*}\right) \subset\left\{x^{*} \in \mathbb{R}^{n} \mid \exists p^{*} \in \mathbb{R}^{p} \text { with }\left(x^{*},-y^{*}\right) \in \partial\left\langle p^{*}, q\right\rangle(\bar{x}, \bar{y})+D^{*} Q(\bar{x}, \bar{y}, \bar{p})\left(p^{*}\right)\right\}
$$

established in [10, Theorem 4.1] assuming that (3.6) has only the trivial solution.

Note that the triviality of solutions to the adjoint generalized equation (3.6) ensures the necessary optimality conditions in normal form (with $z^{*} \neq 0$ ) and can be treated as an appropriate counterpart of the classical Mangasarian-Fromovitz constraint qualification for general optimization problems with equilibrium constraints.

In EPECs and MPECs that are the most interesting for the theory and applications, equilibrium/variational constraints are defined via first-order subdifferentials of extended-real-valued functions; see, e.g., the above cases of variational inequalities and complementarity problems, as well as the so-called hemivariational inequalities usually formulated via Clarke's generalized gradient of Lipschitzian functions. Let us consider a broad class of multiobjective optimization problems with equilibrium constraints, where the multivalued part of the generalized equation (3.5) is given by the basic subdifferential (2.3) of the composition $\partial(\psi \circ g)$ involving an extended-real-valued function $\psi$ and a mapping $g$. Following mechanical terminology, we call the function $\phi:=\psi \circ g$ under the subdifferential operator in the generalized equation by potential.

The first-order necessary optimality conditions obtained below for EPECs governed by variational systems with composite potentials are expressed in terms of the second-order construction (2.4) for extended-real-valued functions. First we consider the case of parameter-independent potentials particularly related to variational and hemivariational inequalities labelled ny HVI. Again we present the results only for Lipschitzian mappings $f$ in multiobjective/equilibrium criteria referring the reader to [13] for more general settings.

Theorem 3.4 (optimality conditions for EPECs governed by HVI with parameterindependent potentials). Let $(\bar{x}, \bar{y})$ be locally $(f, \Theta)$-optimal subject to

$$
0 \in q(x, y)+\partial(\psi \circ g)(y)
$$

where $f: \mathbb{R}^{n} \times \mathbb{R}^{m} \rightarrow \mathbb{R}^{d}$ is Lipschitz continuous around $(\bar{x}, \bar{y}), \Theta$ is locally closed around $0 \in \Theta$, $q: \mathbb{R}^{n} \times \mathbb{R}^{m} \rightarrow \mathbb{R}^{m}$ is strictly differentiable at $(\bar{x}, \bar{y})$ with the partial Jacobian $\nabla_{x} q(\bar{x}, \bar{y})$ of full rank, $g: \mathbb{R}^{m} \rightarrow \mathbb{R}^{s}$ is $\mathcal{C}^{2}$ around $\bar{y}$ with the Jacobian $\nabla g(\bar{y})$ of full rank, and $\psi: \mathbb{R}^{s} \rightarrow \overline{\mathbb{R}}$. Suppose that $\operatorname{gph} \partial \psi$ is closed around $(\bar{w}, \bar{v})$, where $\bar{w}:=g(\bar{y})$ and $\bar{v} \in \mathbb{R}^{s}$ is a unique vector satisfying

$$
-q(\bar{x}, \bar{y})=\nabla g(\bar{y})^{*} \bar{v}, \quad \bar{v} \in \partial \psi(\bar{w})
$$

the latter assumption is automatic if $\psi$ is either convex or continuous around $\bar{w}$. Then there are $z^{*} \in N(0 ; \Theta) \backslash\{0\}$ and $u \in \mathbb{R}^{m}$ such that

$$
0 \in \partial\left\langle z^{*}, f\right\rangle(\bar{x}, \bar{y})+\nabla q(\bar{x}, \bar{y})^{*} u+\left(0, \nabla^{2}\langle\bar{v}, g\rangle(\bar{y})^{*} u+\nabla g(\bar{y})^{*} \partial^{2} \psi(\bar{w}, \bar{v})(\nabla g(\bar{y}) u)\right) .
$$


Proof. Let us derive this result from Theorem 3.3 with $Q(y)=\partial(\psi \circ g)(y)$ by using the second-order subdifferential calculus. Indeed, by (2.4) we have

$$
D^{*} Q(\bar{y}, \bar{p})(u)=\partial^{2}(\psi \circ g)(\bar{y}, \bar{p})(u) \text { with } \vec{p}:=-q(\bar{x}, \bar{y})
$$

Observe that the qualification condition (3.6) holds due the full rank assumption on $\nabla_{x} q(\bar{x}, \bar{y})$ and the independence of $Q$ on the parameter $x$. Employing now the second-order subdifferential chain rule in the case of the surjective (full rank) derivative $\nabla g(\bar{y})$, one gets the equality

$$
\partial^{2}(\psi \circ g)(\bar{y}, \bar{p})(u)=\nabla^{2}\langle\bar{v}, g\rangle(\bar{y})^{*} u+\nabla g(\bar{y})^{*} \partial^{2} \psi(\bar{w}, \bar{v})(\nabla g(\bar{y}) u)
$$

see [14, Theorem 3.4(i) and Remark 3.5] and [9, Theorem 4.1]. This yields the optimality condition (3.7) due to the one in Theorem 3.3.

Next we consider EPECs of the above type but with parameter-dependent potentials, which belong to a class of functions especially important in composite optimization. Recall [21] that $\varphi: \mathbb{R}^{n} \rightarrow \overline{\mathbb{R}}$ is strongly amenable at $\bar{x}$ if there is a neighborhood $U$ of $\bar{x}$ on which $\varphi$ can be represented in the composition form $\varphi=\psi \circ g$ with a $\mathcal{C}^{2}$ mapping $g: U \rightarrow \mathbb{R}^{m}$ and a proper lower semicontinuous convex function $\psi: \mathbb{R}^{m} \rightarrow \overline{\mathbb{R}}$ satisfying the first-order qualification condition $\partial^{\infty} \psi(g(\bar{x})) \cap \operatorname{ker} \nabla g(\bar{x})^{*}=\{0\}$. We refer the reader to [21] for the broad range of calculus and applications of strongly amenable functions. The following theorem gives necessary optimality conditions for EPECs governed by variational systems with strongly amenable potentials that may depend on parameters.

Theorem 3.5 (optimality conditions for EPECs with parameter-dependent amenable potentials). Let $(\bar{x}, \bar{y})$ be locally $(f, \Theta)$-optimal subject to

$$
0 \in q(x, y)+\partial(\psi \circ g)(x, y)
$$

where $f: \mathbb{R}^{n} \times \mathbb{R}^{m} \rightarrow \mathbb{R}^{d}$ and $\Theta \subset \mathbb{R}^{d}$ are the same as in the previous theorem, where $q: \mathbb{R}^{n} \times \mathbb{R}^{m} \rightarrow$ $\mathbb{R}^{n} \times \mathbb{R}^{m}$ is Lipschitz continuous around $(\bar{x}, \bar{y})$, and where the potential in (3.8) is strongly amenable at $(\bar{x}, \bar{y})$. Denote $\bar{p}:=-q(\bar{x}, \bar{y}) \in \partial(\psi \circ g), \bar{w}:=g(\bar{x}, \bar{y})$,

$$
M(\bar{x}, \bar{y}):=\left\{\bar{v} \in \mathbb{R}^{s} \mid \bar{v} \in \partial \psi(\bar{w}), \quad \nabla g(\bar{x}, \bar{y})^{*} \bar{v}=\bar{p}\right\}
$$

and impose the second-order qualification conditions:

$$
\begin{gathered}
\partial^{2} \psi(\bar{w}, \bar{v})(0) \cap \operatorname{ker} \nabla g(\bar{x}, \bar{y})^{*}=\{0\} \text { for all } \bar{v} \in M(\bar{x}, \bar{y}) \text { and } \\
{\left[0 \in \partial\langle u, q\rangle(\bar{x}, \bar{y})+\bigcup_{\bar{v} \in M(\bar{x}, \bar{y})}\left[\nabla^{2}\langle\bar{v}, g\rangle(\bar{x}, \bar{y})(u)+\nabla g(\bar{x}, \bar{y})^{*} \partial^{2} \psi(\bar{w}, \bar{v})(\nabla g(\bar{x}, \bar{y}) u)\right] \Longrightarrow u=0 .\right.}
\end{gathered}
$$

Then there are $z^{*} \in N(0 ; \Theta) \backslash\{0\}$ and $u \in \mathbb{R}^{n} \times \mathbb{R}^{m}$ satisfying

$0 \in \partial\left\langle z^{*}, f\right\rangle(\bar{x}, \bar{y})+\partial\langle u, q\rangle(\bar{x}, \bar{y})+\bigcup_{\bar{v} \in M(\bar{x}, \bar{y})}\left[\nabla^{2}\langle\bar{v}, g\rangle(\bar{x}, \bar{y})(u)+\nabla g(\bar{x}, \bar{y})^{*} \partial^{2} \psi(\bar{w}, \bar{v})(\nabla g(\bar{x}, \bar{y}) u)\right]$. 
Proof. Similarly to the proof of the previous result, we take $Q(x, y)=\partial(\psi \circ g)(x, y)$ in Theorem 3.3 and have

$$
D^{*} Q(\bar{x}, \bar{y}, \bar{p})(u)=\partial^{2}(\psi \circ g)(\bar{x}, \bar{y}, \bar{p})(u) \text { with } \bar{p}:=-q(\bar{x}, \bar{y})
$$

Then the second-order subdifferential chain rule of [9, Corollary 4.3] applied to the strongly amenable potential $\psi \circ g$ gives the inclusion

$$
\partial^{2}(\psi \circ g)(\bar{x}, \bar{y}, \bar{p})(u) \subset \bigcup_{\bar{v} \in M(\bar{x}, \bar{y})}\left[\nabla^{2}\langle\bar{v}, g\rangle(\bar{x}, \bar{y})(u)+\nabla g(\bar{x}, \bar{y})^{*} \partial^{2} \psi(\bar{w}, \bar{v})(\nabla g(\bar{x}, \bar{y}) u)\right]
$$

subject to the second-order qualification condition (3.5). Substituting this into the adjoint generalized equation (3.6) and the optimality condition of Theorem 3.3, we arrive at the required conclusion of this theorem under the assumptions made.

Note that, besides the case of the full rank Jacobian $\nabla g(\bar{x}, \bar{y})$, the second-order qualification condition (3.5) is automatic when $\psi$ is a $\mathcal{C}^{1,1}$ function around $\bar{w}=g(\bar{x}, \bar{y})$; more generally, when the first-order subdifferential mapping $\partial \psi$ has Aubin's Lipschitz-like ("pseudo-Lipschitzian") property around $(\bar{w}, \bar{p})$. This follows from the coderivative criterion for the Aubin property; see, e.g., [10] and [21, Theorem 9.40] for more details and references.

Another interesting class of composite variational systems governing equilibrium constraints (3.5) in MPECs and EPECs are those with the so-called composite fields of the type

$$
Q(x, y)=(\partial \psi \circ g)(x, y)
$$

involving the basic first-order subdifferential (2.3). Systems of such types frequently arise, i.e., in the modeling of mechanical and economic equilibria and cover, in particular, parameter-dependent implicit complementarity problems [16]: given $x \in \mathbb{R}^{n}$, find $y \in \mathbb{R}^{m}$ satisfying

$$
f(x, y) \geq 0, \quad y-g(x, y) \geq 0, \quad\langle f(x, y), y-g(x, y)\rangle=0 .
$$

Our next result gives necessary optimality conditions for EPECs (and more general multiobjective optimization) with equilibrium constraints of this type. For simplicity we consider only a special case of smooth vector functions $q, g$ in defining equilibrium constraints. More general cases, including infinite-dimensional problems, are studied in [13].

Theorem 3.6 (optimality conditions for EPECs with composite fields). Let $(\bar{x}, \bar{y})$ be locally $(f, \Theta)$-optimal subject to

$$
0 \in q(x, y)+(\partial \psi \circ g)(x, y)
$$

where $f: \mathbb{R}^{n} \times \mathbb{R}^{m} \rightarrow \mathbb{R}^{d}$ is Lipschitz continuous around $(\bar{x}, \bar{y}), \Theta$ is locally closed around $0 \in$ $\Theta$, both $q: \mathbb{R}^{n} \times \mathbb{R}^{m} \rightarrow \mathbb{R}^{s}$ and $g: \mathbb{R}^{n} \times \mathbb{R}^{m} \rightarrow \mathbb{R}^{s}$ are strictly differentiable at $(\bar{x}, \bar{y})$, and the subdifferential graph gph $\partial \psi$ is locally closed around $(\bar{w}, \bar{p})$ with $\bar{w}:=g(\bar{x}, \bar{y})$ and $\vec{p}:=-q(\bar{x}, \bar{y})$; the latter is automatics for continuous and for amenable functions $\psi: \mathbb{R}^{s} \rightarrow \overline{\mathbb{R}}$. Assume the fulfilment of the constraint qualifications

$$
\partial^{2} \psi(\bar{w}, \bar{p})(0) \cap \operatorname{ker} \nabla g(\bar{x}, \bar{y})^{*}=\{0\} \quad \text { and }
$$




$$
\left[0 \in \nabla q(\bar{x}, \bar{y})^{*} u+\nabla g(\bar{x}, \bar{y})^{*} \partial^{2} \psi(\bar{w}, \bar{p})(u)\right] \Longrightarrow u=0 .
$$

Then there are $z^{*} \in N(0 ; \Theta) \backslash\{0\}$ and $u \in \mathbb{R}^{s}$ satisfying

$$
0 \in \partial\left\langle z^{*}, f\right\rangle(\bar{x}, \bar{y})+\nabla q(\bar{x}, \bar{y})^{*} u+\nabla g(\bar{x}, \bar{y})^{*} \partial^{2} \psi(\bar{w}, \bar{p})(u) \text {. }
$$

Proof. We employ again Theorem 3.3 with $Q(x, y)=(\partial \psi \circ g)(x, y)$, in which case

$$
D^{*} Q(\bar{x}, \bar{y}, \bar{p})(u)=D^{*}(\partial \psi \circ g)(\bar{x}, \bar{y}, \bar{p})(u) \text { for all } u \in \mathbb{R}^{s}
$$

Using now the coderivative chain rule

$$
D^{*}(\partial \psi \circ g)(\bar{x}, \bar{y}, \bar{p}) \subset \nabla g(\bar{x}, \bar{y})^{*} \partial^{2} \psi(\bar{w}, \bar{p})
$$

valid by (2.4) under the first qualification condition of the theorem (see [7, Corollary 5.4] and [21, Theorem 10.37]) and substituting it into the qualification and optimality conditions of Theorem 3.3 with the specified form of $Q(x, y)$, we arrive at (3.10) under the assumption made.

\section{Necessary Conditions for EPECs via Closed Preference Rela- tions}

In the concluding section of the paper we consider multiobjective optimization problems with equilibrium constraints, where "minimization" of vector functions $f: \mathbb{R}^{n} \times \mathbb{R}^{m} \rightarrow \mathbb{R}^{d}$ is conducted with respect to some closed preference relations. First recall the corresponding definitions; cf. [15]. Given a subset $Z \subset \mathbb{R}^{d} \times \mathbb{R}^{d}$, we say that $z_{1}$ is preferred to $z_{2}$ and write $z_{1} \prec z_{2}$ if $\left(z_{1}, z_{2}\right) \in Z$. A preference $\prec$ is nonreflexive if the corresponding set $Z$ does not contain the diagonal $(z, z)$. In the sequel we consider nonreflexive preference relations satisfying the following requirements.

Definition 4.1 (closed preference relations). Let

$$
\mathcal{L}(z):=\left\{u \in \mathbb{R}^{d} \mid u \prec z\right\}
$$

be a LEVEL SET at $z \in \mathbb{R}^{d}$ with respect to the given preference $\prec$. We say that $\prec$ is LOCALLY SATIATED around $\bar{z}$ if $z \in \mathrm{cl} \mathcal{L}(z)$ for all $z$ in some neighborhood of $\bar{z}$. Furthermore, $\prec$ is ALMOST TRANSITIVE on $\mathbb{R}^{d}$ provided that for all $u \prec z$ and $v \in \operatorname{cl} \mathcal{L}(u)$ one has $v \prec z$. The preference relation $\prec$ is called CLOSED around $\bar{z}$ if it is locally satiated and almost transitive simultaneously.

Note that, while the local satiation property definitely holds for any reasonable preference, the almost transitivity requirement may be violated for some natural preferences important in applications, in particular, for those related to the $(f, \Theta)$-optimality in Definition 3.1. Indeed, consider the case of the so-called generalized Pareto preference induced by a closed cone $\Theta \subset Z$ such that $z_{1} \prec z_{2}$ if and only if $z_{1}-z_{2} \in \Theta$ and $z_{1} \neq z_{2}$. This is, of course, a particular case of Definition 3.1. It is not difficult to check [1] that the generalized Pareto preference is almost transitive if and only if $\Theta$ is convex and pointed, i.e., $\Theta \cap(-\Theta)=\{0\}$. In particular, the almost transitivity condition may fail to fulfill [1] for the following important case of generalized Pareto preferences, and hence in the setting of Definition 3.1. 
Example 4.2 (lexicographical order). Let $\prec$ be a preference on $\mathbb{R}^{d}$ defined by the lexicographical order, i.e., $u \prec v$ if there is an integer $j \in\{0, \ldots, d-1\}$ such that $u_{i}=v_{i}$ for $i=1, \ldots, j$ and $u_{j+1}<v_{j+1}$ for the corresponding components of the vectors $u, v \in \mathbb{R}^{d}$. Then this preference is locally satiated but not almost transitive on $\mathbb{R}^{d}$.

Proof. It is easy to see that

$$
\operatorname{cl} \mathcal{L}(u)=\left\{v=\left(v_{1}, \ldots, v_{d}\right) \in \mathbb{R}^{d} \mid v_{1} \leq u_{1}\right\}
$$

for the lexicographical preference $\prec$, which shows that it is locally satiated on $\mathbb{R}^{d}$. On the other hand, this preference is generated by the convex cone $\Theta:=\left\{\left(z_{1}, \ldots, z_{d}\right) \in \mathbb{R}^{d} \mid z_{1} \leq 0\right\}$, which is not pointed, and thus the almost transitivity property does not hold by the previous discussion. To illustrate this specifically, let us consider the vectors

$$
z:=(0,0,1, \ldots, 0), \quad u:=(0, \ldots, 0), \quad v:=(0,1,1,0, \ldots, 0)
$$

in $\mathbb{R}^{d}$ and the sequence $v_{k}:=(-1 / k, 1,1,0, \ldots, 0) \rightarrow v$ as $k \rightarrow \infty$. Then $u \prec z, v_{k} \prec u$, but $v \nprec z$ while $v \in \operatorname{cl} \mathcal{L}(u)$.

On the other hand, the notion of multiobjective optimization from Definition 4.1 may cover preference relations that are not described by the way of Definition 3.1. Indeed, the concept of optimality from Definition 4.1 relates to nonlinear transformations of sets in extremal systems instead of their translations as in Definition 3.1; see [15] for more discussions and examples. To deal with such transformed sets in extremal systems, we use the version of the extremal principle for set-valued mappings (moving sets) established in [15]. Going in this direction, we derive now new necessary optimality conditions for multiobjective problems with equilibrium constraints, where the multiobjective optimality is understood in the sense of closed preference relations. Due the above discussion, these results are independent of those from Section 3.

To formulate optimality conditions for EPECs with respect to closed preferences, we need the following construction of the extended normal cone $\tilde{N}(\bar{z} ; \Omega(\bar{x}))$ to a moving set $\Omega: \mathbb{R}^{n} \rightarrow \mathbb{R}^{d}$ at $(\bar{x}, \bar{z}) \in \operatorname{gph} \Omega$ described by

$$
\tilde{N}(\bar{z} ; \Omega(\bar{x})):=\operatorname{Limsup}_{(x, z) \stackrel{\operatorname{sph} \Omega}{\rightarrow}(\bar{x}, \bar{z})} \widehat{N}(z ; \Omega(x))
$$

via the Fréchet normal cone from Section 2. It follows from the review in Section 2 that the extended normal cone (4.1) agrees with the basic one in (2.1) for fixed sets $\Omega(x) \equiv \Omega$. This also happens for a large class of moving sets $\Omega(\cdot)$ but may be violated in some important settings; see [15] for more details and discussions.

Let us first present necessary optimality conditions for multiobjective problems with respect to closed preferences in the case of equilibrium constraints given in the abstract geometric form $y \in S(x)$. For simplicity we consider only Lipschitzian objectives referring the reader to [13] for more general problems including those in infinite dimensions.

Theorem 4.3 (optimality conditions for abstract EPECs with closed preferences). Let $(\bar{x}, \bar{y})$ be a local optimal solution to the multiobjective problem:

$$
\text { minimize } f(x, y) \text { with respect to } \prec \text { subject to } y \in S(x) \text {, }
$$


where $f: \mathbb{R}^{n} \times \mathbb{R}^{m} \rightarrow \mathbb{R}^{d}$ is Lipschitz continuous around $(\bar{x}, \bar{y})$ with $\bar{z}:=f(\bar{x}, \bar{y})$, where $S: \mathbb{R}^{n} \rightrightarrows \mathbb{R}^{m}$ is closed-graph around $(\bar{x}, \bar{y})$, and where the preference $\prec$ is closed. Then one has

$$
0 \in \partial\left\langle z^{*}, f\right\rangle(\bar{x}, \bar{y})+N((\bar{x}, y) ; \operatorname{gph} S) \text { for some } z^{*} \in \tilde{N}(\bar{z} ; \operatorname{cl} \mathcal{L}(\bar{z})) \backslash\{0\} .
$$

Proof. Check that $(\bar{x}, \bar{y}, \bar{z})$ is a locally extremal point at $(\bar{z}, 0)$ in the sense of [15, Definition 3.3] of the system of closed-valued multifunctions $S_{i}: M_{i} \rightrightarrows \mathbb{R}^{n} \times \mathbb{R}^{m} \times \mathbb{R}^{d}, i=1,2$, defined by

$$
\begin{aligned}
& S_{1}(z):=\operatorname{gph} S \times \operatorname{cl} \mathcal{L}(z) \text { with } M_{1}:=\mathcal{L}(\bar{z}) \cup\{\bar{z}\} \\
& S_{2} \equiv \operatorname{gph} f \text { with } M_{2}:=\{0\} .
\end{aligned}
$$

Indeed, one easily gets $(\bar{x}, \bar{y}, \bar{z}) \in S_{1}(\bar{z}) \cap S_{2}$ due to the local satiation property of $\prec$. Assuming now that $(\bar{x}, \bar{y}, \bar{z})$ is not a locally extremal point of $(4.3)$ at $(\bar{z}, 0)$, we find, given any neighborhood $U$ of $(\bar{x}, \bar{y}, \bar{z})$, a point $z$ from the level set $\mathcal{L}(\bar{z})$ close to $\bar{z}$ but not equal to the latter by the preference nonreflexivity, for which

$$
[\operatorname{gph} S \times \operatorname{cl} \mathcal{L}(z)] \cap \operatorname{gph} f \cap U \neq \emptyset .
$$

This yields the existence of $(x, y)$ near $(\bar{x}, \bar{y})$ with $y \in S(x)$ and $f(x, y) \in \operatorname{cl} \mathcal{L}(z)$. Hence $f(x, y) \prec$ $f(\bar{x}, \bar{y})$ by the almost transitivity property of $\prec$, and (4.4) contradicts the local optimality of $(\bar{x}, \bar{y})$ in the constrained multiobjective problem under consideration. Thus $(\bar{x}, \bar{y}, \bar{z})$ is a locally extremal point of the system $\left\{S_{1}, S_{2}\right\}$ in (4.3). Employing now to this systems the extremal principle for multifunction from [15, Theorem 4.7] and taking into account the scalarization formula from Section 2, we arrive at (4.2) and conclude the proof of the theorem.

Next let us consider necessary conditions with respect to closed preference relations for EPECs involving equilibrium constraints governed by parametric variational systems (3.5).

Theorem 4.4 (optimality conditions for EPECs with closed preferences and constraints governed by variational systems). Let $(\bar{x}, \bar{y})$ be a local optimal solution to the multiobjective optimization problem:

$$
\text { minimize } f(x, y) \text { with respect to } \prec \text { subject to } 0 \in q(x, y)+Q(x, y) \text {, }
$$

where $f: \mathbb{R}^{n} \times \mathbb{R}^{m} \rightarrow \mathbb{R}^{d}, q: \mathbb{R}^{n} \times \mathbb{R}^{m} \rightarrow \mathbb{R}^{p}, Q: \mathbb{R}^{n} \times \mathbb{R}^{m} \rightrightarrows \mathbb{R}^{p}$, and where $\prec$ is a closed preference relation on $\mathbb{R}^{d}$. Assume that both $f$ and $q$ are locally Lipschitzian around $(\bar{x}, \bar{y})$, that $Q$ is closed-graph around $(\bar{x}, \bar{y}, \bar{p})$ with $\bar{p}:=-q(\bar{x}, \bar{y})$, and that the qualification condition

$$
\left[0 \in \partial\left\langle p^{*}, q\right\rangle(\bar{x}, \bar{y})+D^{*} Q(\bar{x}, \bar{y}, \bar{p})\left(p^{*}\right)\right] \Longrightarrow p^{*}=0
$$

is fulfilled. Then there are $z^{*} \in \tilde{N}\left((\bar{z} ; \operatorname{cl} \mathcal{L}(\bar{z})) \backslash\{0\}\right.$ and $p^{*} \in \mathbb{R}^{p}$ satisfying

$$
0 \in \partial\left\langle z^{*}, f\right\rangle(\bar{x}, \bar{y})+\partial\left\langle p^{*}, q\right\rangle(\bar{x}, \bar{y})+D^{*} Q(\bar{x}, \bar{y}, \bar{p})\left(p^{*}\right) .
$$

Proof. This follows from Theorem 4.3 with the equilibrium map

$$
S(x):=\left\{y \in \mathbb{R}^{m} \mid 0 \in q(x, y)+Q(x, y)\right\}
$$


due to the coderivative inclusion

$$
D^{*} S(\bar{x}, \bar{y})\left(y^{*}\right) \subset\left\{x^{*} \in \mathbb{R}^{n} \mid \exists p^{*} \in \mathbb{R}^{p} \text { with }\left(x^{*},-y^{*}\right) \in \partial\left\langle p^{*}, q\right\rangle(\bar{x}, \bar{y})+D^{*} Q(\bar{x}, \bar{y}, \bar{p})\left(p^{*}\right)\right\}
$$

derived in [10, Theorem 4.1] under the qualification condition (4.5).

Similarly to Theorems 3.3-3.5, we can derive from Theorem 4.4 necessary optimality conditions for multiobjective optimization problems, particularly for EPECs, with respect to closed preferences and the specific equilibrium constraints considered in Section 3.

In conclusion we observe that EPECs considered in this paper are intrinsically nonsmooth, even in the simplest settings of equilibrium constraints governed by parameter-dependent variational inequalities and complementarity conditions. In particular, for models studied in Theorems 3.4-3.6 this relates to the nonsmoothness of the potential $\psi$, which is actually the indicator (extended-realvalued) function of a convex set for standard cases of complementarity and variational inequality constraints. Practical implementations of the optimality conditions obtained in the mentioned theorems require computing/estimating the second-order subdifferentials for favorable classes of nonsmooth functions $\psi$ involved in these models. Efficient evaluations of the second-order subdifferentials and their applications to some problems with equilibrium constraints are given in $[2,14,16,23]$ and the references therein. Such evaluations and the results obtained in this paper allow us to specify classes of MPECs and EPECs that can be effectively handled by generalized differential tools of variational analysis.

\section{References}

[1] K.J. Eisenhart (2003), Multiobjective Optimal Control Problems with Endpoint and State Constraints. Ph.D. Dissertation, Western Michigan University, Kalamazoo, MI.

[2] A.L. Dontchev and R.T. Rockafellar (1996). Characterization of strong regularity for variational inequalities over polyhedral convex sets. SIAM J. Optim., 7, 1087-1105.

[3] F. Faccinei and J.-S. Pang (2003). Finite-Dimensional Variational Inequalities and Complementarity Problems, published in two volumes. Springer, New York.

[4] X. Hu, D. Ralph, E.K. Ralph, P. Bardsley and M.C. Ferris (2002). The effect of transmission capacities on competition in deregulated electricity markets. Preprint.

[5] Z.-Q. Luo, J.-S. Pang and D. Ralph (1996). Mathematical Programs with Equilibrium Constraints. Cambridge University Press, Cambridge, UK.

[6] B.S. Mordukhovich (1988). Approximation Methods in Problems of Optimization and Control. Nauka, Moscow.

[7] B.S. Mordukhovich (1994). Generalized differential calculus for nonsmooth and set-valued mappings. J. Math. Anal. Appl., 183, 250-288.

[8] B.S. Mordukhovich (2001). The extremal principle and its applications to optimization and economics. In: A. Rubinov and B. Glover (Eds.), Optimization and Related Topics, pp. 343-369 in Applied Optimization Series, 47. Kluwer Academic Publishers, Dordrecht, The Netherlands. 
[9] B.S. Mordukhovich (2002). Calculus of second-order subdifferentials in infinite dimensions. Control and Cybernetics, 31, 557-574.

[10] B.S. Mordukhovich (2003). Coderivative analysis of variational systems. J. Global Optim., to appear.

[11] B.S. Mordukhovich (2003). Necessary conditions in nonsmooth minimization via lower and upper subgradients. Set-Valued Anal., to appear.

[12] B.S. Mordukhovich (2003). Optimization and equilibrium problems with equilibrium constraints. OPSEARCH, to appear.

[13] B.S. Mordukhovich (2004), Variational Analysis in Infinite Dimensions. Springer, to appear.

[14] B.S. Mordukhovich and J.V. Outrata (2001). On second-order subdifferentials and their applications. SIAM J. Optim., 12, 139-169.

[15] B.S. Mordukhovich, J.S. Treiman and Q.J. Zhu (2003). An extended extremal principle with applications to multiobjective optimization. SIAM J. Optim., to appear.

[16] J.V. Outrata (2000). A general mathematical program with equilibrium constraints. SIAM J. Control Optim., 38, 1623-1638.

[17] J.V. Outrata (2003). A note on a class of equilibrium problems with equilibrium constraints. Kibernetika, to appear.

[18] J.V. Outrara, M. Kočvara and J. Zowe (1998). Nonsmooth Approach to Optimization Problems with Equilibrium Constraints. Kluwer Academic Publisher, Dordrecht, The Netherlands.

[19] S.M. Robinson (1979). Generalized solutions and their solutions, part 1: basic theory. Math. Programming Study, 10, 128-141.

[20] S.M. Robinson (2003). Locallized normal maps and the stability of variational conditions. Set-Valued Anal., to appear.

[21] R.T. Rockafellar and R.J.-B. Wets (1998). Variational Analysis. Springer, Berlin.

[22] H. Scheel and S. Scholtes (2000). Mathematical programs with equilibrium constraints:stationarity, optimality and sensitivity. Mathematics of Operations Research, 25, 1-22.

[23] J.J. Ye (2000). Constraint qualifications and necessary optimality conditions for optimization problems with variational inequality constrainys. SIAM J. Optim., 10, 943-962. 